\title{
Probiotics, Nutrition, and the Small Intestine
}

\author{
Taylor C. Judkins ${ }^{1} \cdot$ Douglas L. Archer ${ }^{1} \cdot$ Dean C. Kramer $^{2} \cdot$ Rebecca J. Solch $^{1}$
}

Published online: 13 January 2020

(C) The Author(s) 2020

\begin{abstract}
Purpose of Review Probiotics are promising remedial treatments for symptoms of small intestine (SI) diseases and promoters of overall good health. Probiotics play an important role in supporting a healthy SI microbiome (eubiosis), and in preventing establishment of unhealthy microbiota. SI eubiosis promotes optimal nutrient uptake, and optimal nutritional status maintains a healthy SI, reducing the likelihood of SI diseases. It is important to understand the advantages and limitations of probiotic therapies.

Recent Findings Microbial dysbiosis decreases the capacity of the small bowel to utilize and absorb dietary compounds. In some studies, probiotic supplements containing lactic acid bacteria and Bifidobacterium have been demonstrated effective in supporting beneficial microbes in the SI while improving barrier integrity and reducing nutrient malabsorption and SI diseaserelated pathology.

Summary Strain-specific probiotic therapy may be a natural and effective approach to restoring SI barrier integrity and eubiosis, resulting in improved nutrient absorption and better health, including reducing the incidence of and severity of SI diseases.
\end{abstract}

Keywords Small intestine $\cdot$ Probiotics $\cdot$ Nutrition $\cdot$ Microbiota $\cdot$ Dysbiosis, intestinal permeability

\section{Introduction}

The preponderance of nutrient absorption occurs in the small intestine (SI), and diseases affecting the SI may therefore disrupt nutrient absorption. Malnutrition occurs when adequate amounts of single or multiple nutrients cannot gain entry to the body compartment or gain entry in excessive or unbalanced amounts. This can be the result of SI disease pathology, or dysbiosis of the normal SI microbial flora, either of which may alter the structure and permeability of the SI epithelial barrier. Probiotics are live microorganisms that, when ingested in adequate amounts, confer a health benefit to the host [1]. Probiotics are generally regulated as either dietary supplements or medical foods (e.g., Visbiome $\AA$, a multi-strain probiotic formerly called VSL\#3) in the USA. Most probiotics currently available are lactic acid bacteria (LAB), and Bifidobacterium

This article is part of the Topical Collection on Small Intestine

Rebecca J. Solch

rsolch@ufl.edu

1 Food Science and Human Nutrition Department, University of Florida, 572 Newell Dr., Gainesville, FL 32611, USA

2 Gainesville, USA spp., and certain yeasts such as Saccharomyces boulardii [2], which have a long history of safe use and are legally "generally recognized as safe" (GRAS). The effects of probiotics, and moreover studies on the microbial composition of the SI flora, have been hampered by limitations on access, as SI epithelial biopsies or aspirates via naso-ileal catheters are invasive procedures. Therefore, most microbiome analyses are conducted on stool which is influenced heavily by colonic microbiota. Nonetheless, recent animal studies and human clinical trials suggest that probiotics can have a restorative effect on gut integrity and nutrient uptake via promoting eubiosis in the SI.

\section{Small Intestine}

\section{Characteristics}

The SI, comprised of the duodenum, jejunum, and ileum, is the major site of macro- and micronutrient digestion and absorption. Digestion is accomplished through a mixture of digestive enzymes (pancreatic lipases, SI brush-border disaccharidases, etc.) as well as other secretions (i.e., bile salts and bicarbonate) active in digestive processes. Plicae circularis, transverse folds of submucosa covered by mucosa 
predominantly in the duodenum and proximal jejunum, are covered by villi and microvilli to increase the surface area of the SI and optimize nutrient absorption.

The gastrointestinal (GI) tract is lined with the mucosal epithelium to act as a direct barrier between the environment and host. This intestinal barrier contains various components, such as commensal gut microbiota, mucus layer, antimicrobial peptides (AMPs), and junctional complexes (i.e., tight junctions (TJs), adherens junctions, and desmosomes). These dynamic components work together to maintain normal barrier integrity [3]. Permeability of the barrier can be increased through direct damage to the epithelial mucosa or changes to other components via dysbiosis, diet, or inflammation [4].

The duodenum, jejunum, and ileum experience unique luminal environmental factors that can change each section's microbial abundance. On average, the duodenum and jejunum contain up to $10^{3}-10^{4}$ bacteria/mL followed by an increase to $10^{8}$ bacteria $/ \mathrm{mL}$ in the ileum. While the concentration of bacteria increases along the GI tract, in comparison, it is much lower than the typical concentration of the colon $\left(10^{11}\right.$ bacte$\mathrm{ria} / \mathrm{mL}$ ) [5].

\section{Modulators of SI Microbiota}

SI microbiota abundance and composition can be modulated by oxygen availability, $\mathrm{pH}$, transit time, AMPs, and intake of probiotics. Oxygen availability, on average, decreases from proximal to distal SI and microniches in the lumen create environments for aerobes and strict anaerobes alike to survive and metabolize.

The $\mathrm{pH}$ of SI regions and transit time of food content contribute to the changes in microbial density. The median $\mathrm{pH}$ of the proximal intestine is 6.7 with an increase to 7.5 in the terminal ileum [6]. Acidic chyme passes from the stomach into the duodenum and stimulates the hormone secretin, which in turn stimulates the liver and pancreas to release bicarbonate into the duodenum, thus increasing $\mathrm{pH}$ and allowing for optimal function of digestive enzymes. The basic $\mathrm{pH}$ within the terminal ileum may create a more favorable environment for SI microbiota to begin degradation of complex carbohydrates, ferment simple carbohydrates, and utilize energy. These processes are time-limited as food content is only in the SI for 2-5 h [7]. Unabsorbed nutrients and fiber enter the colon where they reside for 12-24 h [7], allowing for fermentation of complex carbohydrates and production of short chain fatty acids (SCFAs).

The microbial environment of the SI can also be shaped by AMPs that function as a part of the innate immune system and thus appear in greater amounts during inflammatory events triggered by dysbiosis or disease. In mice, reduced concentrations of cathelicidin-related AMP resulted in increased duodenal inflammation and permeability allowing for translocation of bacteria to the spleen, liver, and pancreas [8]. Normal AMP secretion is important for maintaining a eubiotic environment and healthy SI barrier.

Consumption of probiotics also impacts the microbial environment of the SI. Probiotics can provide $10^{8}-10^{12}$ colony forming units per day [9]. Assuming $10 \%$ survival of $10^{10}$ ingested probiotic bacteria, the relative abundance of ingested bacteria compared with resident bacteria in the SI can be 0.01 to 1 -fold compared with 0.0001 - to 0.00001 -fold in the colon [9]. This suggests that probiotics may have a greater impact in the SI than in the colon. Ingested probiotic bacteria support the SI microbiota through cross-feeding and reducing or inhibiting pathogens [9]. However, ingested probiotics are considered transients, as they do not become integral members of the core microbiota [10]. Difficulties in sampling the human SI microbiota limit our knowledge of the relationship between additional factors (i.e., dietary components, medications, lifestyle) and the SI microbiota. Table 1 lists some of the factors affecting the composition of the SI microbiota.

\section{SI Microbiota Products}

Phyla present in the SI (Firmicutes, Bacteroidetes, and Actinobacteria) have the ability to produce B-vitamins through biosynthesis pathways $[11 \cdot 12]$. It has been estimated that up to $60 \%$ of microbes can produce each of the Bvitamins [11•] used by either the human host or other microbiota. A eubiotic microbiome also produces butyrate through fermentation which helps maintains the SI epithelial barrier integrity, promotes villus development, and dampens excessive inflammation [13-17]. Thus, changes to the SI microbiota composition that directly or indirectly decrease butyrate

Table 1 Factors that may affect and influence the microbial profile of the small intestine

Nutrient availability

Macronutrients

Micronutrients

Fermentation substrates

Antimicrobial peptides

Gut motility

Gastric acid, bile, pancreatic enzymes

Temperature

$\mathrm{pH}$

Resident microbes

Inflammation

Antibiotics or medications

Topography

Mucosal health

Small intestine surgery

Disease state

Probiotics (food and dietary supplements) 
producers can impact nutrient absorption and gut health of individuals.

\section{Probiotics}

Probiotics are of growing interest due to their modulatory effects on markers of human health. Several meta-analyses demonstrate probiotic benefits in modulating symptoms of various GI diseases, such as irritable bowel syndrome (IBS), inflammatory bowel disease (IBD), and Clostridium difficile infections, as well as mood disorders such as depression [18-21]. Additional meta-analyses have been conducted in order to determine if probiotic efficacy is strain- and diseasedependent [22]. There is strong evidence that probiotics are strain-specific in mitigating symptoms associated with individual diseases [23]. When evaluating a probiotic supplement, the specific strain, the disease, and the individual should be considered, as should results of well-designed human clinical trials.

International consensus states that probiotics exert their benefits to the host by (i) interference with pathogenic bacteria by competing for nutrients and adhesion sites, (ii) improvement of the barrier function of the epithelial lining, (iii) immunomodulation, and (iv) influence on other organs of the body through the immune system and neurotransmitter production [1]. Probiotics also increase the production of vital compounds necessary for eubiosis and human health, including SCFAs such as butyrate [24]. Finally, these beneficial microbes also ensure an intestinal environment where optimal nutrient absorption may occur [24].

\section{Probiotics and the Small Intestine}

As stated previously, studying the microbiome of the SI is difficult, as invasive procedures are generally required. Traditional stool samples collected from humans will identify species indigenous to the colon, plus transient bacteria from food, or the oral, esophageal, or SI microbiota. Current understanding of how probiotics influence the SI is largely derived from animal models. Recently, one group administered three probiotic strains, Lactobacillus salivarius G1-1, L. reuteri G85, and L. reuteri G22-2, and an antibiotic control to groups of piglets and examined the ileal mucosa proteomics [25]. Piglets consuming the lactobacillus strains had expression of 32,40 , and 27 proteins that are associated with maintaining the integrity of cell structure, cell stability, and pathogen defenses, respectively. Another group administered L. rhamnosus GG (LGG) prophylactically to pigs prior to a Salmonella Infantis challenge [26••]. LGG taken prophylactically downregulated the $S$. Infantis-induced increase of $\mathrm{CD}^{+}{ }^{+} \mathrm{IFN}^{+} \mathrm{T}$ cells in Peyer's patches and IL-7R $\alpha$ expression in the jejunum, demonstrating a probiotic benefit exerted through the immune system and the complexity of the interactions occurring.
Probiotics, specifically LAB, may protect the SI by increasing microbial diversity, upregulating protein expression involved in homeostasis, and maintaining immune system integrity.

SI rotavirus diarrhea and antibiotic-associated diarrhea are both routinely treated with probiotics, particularly LGG [27]. LGG is further able to mechanically protect the mucosa and inhibit the attachment of certain pathogenic bacteria [27].

\section{Probiotics and Intestinal Permeability}

A healthy intestinal barrier is selectively permeable, permitting passage of essential nutrients and water while restricting absorption of toxins and pathogens [28]. The TJ, the main regulator of paracelluar permeability, is comprised of transmembrane proteins (claudins), scaffolding proteins (zonulin), and regulatory proteins [29]. Chronic disruption to the gut barrier over time may contribute to GI and autoimmune diseases by stimulating an overactive inflammatory response and may decrease nutrient bioavailability [30]. Probiotics are a potential approach to help maintain the intestinal barrier along the entire intestinal tract. In addition to contributing to butyrate production by a healthy, balanced microbiome, probiotics are effective in strengthening TJ proteins and preserving mucosal integrity, and as such also promote optimal nutrient absorption [31].

One study recently examined the effects of $L$. reuteri $\mathrm{LR} 1$ on intestinal permeability of the SI in weaned pigs [32•]. One hundred forty-four weaned pigs were divided into three intervention groups consisting of a control diet or the same diet plus L. reuteri LR1 or antibiotic treatments for 14 days. When compared with pigs on the antibiotic or control diet, those in the probiotic group had increased villus height to crypt depth ratio and increased TJ protein expression in the mucosa of the jejunum and ileum. Another study administered L. reuteri ZJ617 and LGG by oral gavage to mice who were injected with lipopolysaccharide (LPS) to induce barrier dysfunction [33]. LPS administration caused a reduction in abundance of occludin and claudin-3, and both probiotic strains were able to attenuate the reduction. In another investigation, high doses of kanamycin were administered to disturb the intestinal barrier in mice and study the effects of LAB on Peyer's patch cells in the ileum [34]. When compared with mice on the control diet, those receiving LAB had increased expression of zonulin-1 and occludin in the ileal tissue. They also had higher levels of serum immunoglobulin A in Peyer's patch cells, reflecting that Peyer's patches were protected from kanamycin by LAB. Based on evidence for LAB maintaining barrier integrity observed in recent animal studies, similar studies should be undertaken in humans $[35,36]$. The yeast $S$. boulardii has been shown to be very effective in treatment of clinical disorders with associated intestinal barrier disruption in both animal studies and human clinical trials [2]. 
Diseases of the Small Intestine and Nutritional Impacts

\section{Small Intestinal Bacterial Overgrowth}

Small intestinal bacterial overgrowth (SIBO) has been implicated as a cause of chronic diarrhea and nutrient malabsorption. Estimates of prevalence of SIBO vary based on the testing methods used to diagnose this disease, and many testing methods, such as hydrogen breath tests, are imprecise [37]. Functional GI symptoms of SIBO do not correlate with quantitative SI bacterial culture profiles, but do correlate with dysbiosis as defined by $16 \mathrm{~S}$ rRNA sequencing of the SI microbiota [38, 39]. Nutrient malabsorption can range from mild to profound, resulting in weight loss and vitamin deficiencyassociated neuropathies [37].

Persons with SIBO have between $10^{5}$ and $10^{6}$ bacteria $/ \mathrm{mL}$ luminal content, 2 to $3 \log _{10} / \mathrm{mL}$ higher than healthy individuals [40]. The bacterial species contaminating the SI in SIBO patients are commonly identified oropharyngeal and colonic flora, including microaerophilic bacteria such as Streptococcus, Escherichia coli, Staphylococcus, Micrococcus, Klebsiella, and Proteus, and anaerobic bacteria such as Lactobacillus, Bacteroides, Clostridium, Veillonella, Fusobacterium, and Peptostreptococcus [41]. The most commonly prescribed treatment for SIBO is the broad-spectrum antibiotic rifaximin; however, this medication only has a $66.7 \%$ cure rate $[42,43]$. Rifaximin also has the potential to disturb commensal bacterial populations and induce antibiotic-associated diarrhea and C. difficile infections. Therefore, other therapeutic options such as probiotics to mitigate bacterial overgrowth and repopulate the SI with beneficial bacteria are of interest [44]. Efficacy studies of probiotics in treating SIBO have yielded discordant results [45]. A meta-analysis and systematic review concluded that probiotics were effective at SIBO decontamination and symptom relief, but were ineffective in SIBO prevention [45]. It should be noted that consumption of certain probiotic strains (e.g., Bifidobacterium infantis) may increase methane gas levels suggestive of SIBO in response to the lactulose breath test [46].

\section{Irritable Bowel Syndrome}

Discussions about IBS are made difficult by proposed disparate symptomatic subtypes and etiologies [47]. IBS is characterized by abdominal pain associated with altered bowel habits in the form of constipation, diarrhea, or both [48]. SIBO may or may not be present concurrently with IBS. Evidence of a role for SI dysbiosis in IBS is strong, but treatment with probiotics, although yielding promising results, is hampered by not knowing the effectiveness of the specific probiotic strain(s), dose, or necessary duration of treatment [49]. However, treatment with probiotic Bacillus spp. spores reportedly improved measurements of the quality of life of IBS patients, probably owing to modification of the gut microbiota [50]. As with SIBO, altered SI permeability is present in IBS, but only in the diarrhea-predominant subtype [51]. It can safely be concluded that along with permeability changes and associated diarrhea with decreased transit time, nutrient uptake is negatively affected.

\section{Crohn's Disease}

The inflammatory state of Crohn's disease (CD) can affect SI permeability and reduce nutrient absorption, putting individuals at an increased risk of malnutrition. Immunohistochemical analyses of duodenal biopsies from active $\mathrm{CD}$ showed destruction and dilation of TJs compared with controls. This damage coincided with shortening of the microvilli and increased intervilli distance [52]. Damage to the mucosa, through villi blunting, can limit absorptive capabilities of the SI through loss of brush-border enzymes [53] and reduced surface area.

Nutrient absorption is highly dependent on the action of transporters at the apical surface of epithelial cell membranes. Transcriptional analysis of the ileal mucosa of CD individuals revealed alterations in the expression of 62 solute carrier transporters (SLC) and zinc transporters. The majority of the SLC transporters were downregulated, including those important for amino acid transport. Low levels of transporters and metallothioneins important for the absorption, storage, and export into circulation of zinc was also seen in CD mucosa [54.•]. The low expression of the transporters limits the amount of nutrients that enter the enterocyte, ultimately lowering the concentrations in circulation. When the relationship between microbial species and transporter expression was examined by incubating human ileal mucosa with $L$. casei, only partial recovery of SLC transporter expression was shown [54・•]. Although humans are more variable in both ileal microbial composition and physiological processes than an in vitro study, the study provides evidence for a role of intestinal microbiota in $\mathrm{CD}$.

In individuals who have treatment-naïve $\mathrm{CD}$, the SI microbiota is dysbiotic due to a decrease in butyrate producers [55-58]. The genera Bacteroides and Clostridiales are absent in $\mathrm{CD}$ individuals $\left[56^{\bullet}, 58\right]$ and negative associations for $\mathrm{CD}$ severity were found with lower abundance of the genera: Bacteroides, Faecalibacterium, Roseburia, Blautia, Ruminococcus, and Coprococcus [56•], butyrate producers responsive to probiotic support. Decreased butyrate production could contribute to compromised SI barrier integrity, thus affecting nutrient absorption and increasing inflammation and disease severity. In an in vitro model of CD microbiota, the addition of six butyrate producing bacteria to monolayers of intestinal epithelium cells exposed to CD fecal-derived cultures improved epithelial barrier integrity as measured by transepithelial electrical resistance (TEER) and apparent permeability of the paracellular marker Lucifer yellow [59]. 
TEER is a widely accepted quantitative technique to measure the integrity of tight junctions in cell culture models of the intestinal epithelium. Colonization capacity in mucus- and lumen-associated CD microbiota was highest when a mixture of butyrate producers was used [59] suggesting that one species alone may not be able to establish within resident microbiota.

A systematic review of 9 studies found little benefit of probiotics in persons with CD [60]. However, many of these studies focused on the use of Bifidobacterium and Lactobacillus. Interestingly, these genera have been found to be at higher concentrations in gut mucosal biopsies in active CD patients [55]. Future probiotic studies should evaluate the use of combination butyrate producers not currently available as dietary supplement probiotics [61].

\section{Nutrition and the Small Intestine Microbiome}

Persons with SI diseases that demonstrate malabsorption exhibit distinctive microbiota profiles. A pilot study compared duodenal fluid between children recently diagnosed with IBD to healthy controls [62]. Children with IBD had decreases in total microbial counts of Collinsella, Lactobacillus and Bacillus, Firmicutes, Actinobacteria, and Bacteroidetes. This information is of value as patients with IBD are at risk of malabsorption with micronutrient deficiencies, perhaps related to the dysbiosis observed in the SI [63]. The SI microbiome also dictates how a host will digest and absorb dietary compounds, such as lipids, which may lead to over or under nutrition. One study provided a high-fat diet to germ-free (GF) mice and controls housed under standard conditions and found that GF mice had impaired lipid digestion compared with controls, suggesting an important role for microbiota in digestion/absorption [64].

Other studies have also demonstrated that dietary patterns influence the SI microbiota, which in turn may affect health status. In one study, pigs were fed a diet with a standard concentration of protein $(16 \%)$, a diet that was moderately reduced in protein diet $(13 \%)$, or a diet low in protein $(10 \%)$ for 28 days [65]. Ileal samples were obtained at slaughter for microbiota analysis. Ileal bacteria richness decreased as dietary protein was decreased to $10 \%$; however, TJ protein expression was highest in those receiving the $13 \%$ diet. This suggests that a diet that moderately restricted protein intake may actually promote a healthier pattern of ileal bacterial community. Future research may define optimal bacterial communities to promote health and divulge the dietary patterns to build those communities. Other dietary compounds such as sugar substitutes, food additives, and emulsifiers are associated with low microbial diversity and increased inflammation in the SI $[66,67]$. Diets rich in polyphenols, fiber, and whole plant sources, however, are associated with increased biodiversity in fecal samples and the upregulation of commensal bacteria in the microbiome [68]. Unfortunately, typical western diets containing processed foods and acellular nutrients are more bioavailable in the SI [69]. This then provides ample nutrients that fuel adverse changes in microbiota composition of the SI [70]. When discussing nutrition and the SI, an interdependent relationship is observed. Beneficial microbes may allow for the optimal absorption and utilization of dietary nutrients while a proper diet will increase microbial diversity and abundances of valuable species to promote efficient nutrient absorption.

\section{Conclusion}

The SI is the major site of nutrient absorption, and disruption of normal SI function and integrity can lead to nutritional deficiencies and malnutrition [71, 72]. SI microbiota may be a significant contributor in the development of SI diseases such as SIBO, IBS, and CD, and overt or covert malnutrition. Beneficial microbes produce valuable compounds, such as butyrate, which support proper SI structure and physiology needed to optimally harness nutrients. Therefore, the composition of the SI microbiota plays a substantial role in predicting and influencing human health [73].

Probiotics could help maintain a eubiotic environment, correct dysbiosis, and ameliorate nutrient malabsorption issues within the SI. However, the use of probiotics is complicated as characterization of the SI microbiota in healthy adults, and clinical trials to evaluate probiotic efficacy are relatively scarce, likely due to the invasive sampling procedures required to examine SI contents. Future studies could utilize ex vivo models of SI such as enteroids, 3-dimensional organoids derived from SI stem cells to study probiotic interactions with the SI epithelium [74], and explore new technologies such as robotic sampling capsules to harvest SI microbiota. Non-invasive access to SI luminal contents will improve understanding of SI microbiota's profile in health and disease and enable more precise studies on the efficacy of probiotics in the SI. Research is also needed to determine efficacy of specific probiotic strains or combinations of strains in therapeutic applications in the SI.

Until new SI lumen sampling methods are available and verified, the use of biomarkers may be the key to determining the status of the SI microbiota, the SI epithelial barrier integrity, and even nutritional status. For example, blood serum analyses for zonulin and bacterial components such as lipopolysaccharide can allude to $\mathrm{TJ}$ integrity, and specific cytokines and immunoglobulins can reflect overall immune status of the SI [75]. Additionally, measuring sugar output in the urine is a promising technique that allows researchers to compare site-specific intestinal permeability during various interventions [76]. 
Eubiosis in the SI creates a homeostatic environment is which the digestive, immune, and endocrine systems collaborate to ensure proper nutrient absorption and utilization. Nutritional status of persons with SI dysbiosis or SI disease should be taken into consideration and probiotics considered as a therapeutic option.

Acknowledgments We would like to acknowledge that the first authors, Taylor C. Judkins, and Rebecca J. Solch contributed equally to the paper. Authors Archer and Kramer contributed to microbiological and clinical aspects of the paper, respectively.

\section{Compliance with Ethical Standards}

Conflict of Interest The authors declare that they have no conflict of interest.

Human and Animal Rights and Informed Consent This article does not contain any studies with human or animal subjects performed by any of the authors.

Open Access This article is licensed under a Creative Commons Attribution 4.0 International License, which permits use, sharing, adaptation, distribution and reproduction in any medium or format, as long as you give appropriate credit to the original author(s) and the source, provide a link to the Creative Commons licence, and indicate if changes were made. The images or other third party material in this article are included in the article's Creative Commons licence, unless indicated otherwise in a credit line to the material. If material is not included in the article's Creative Commons licence and your intended use is not permitted by statutory regulation or exceeds the permitted use, you will need to obtain permission directly from the copyright holder. To view a copy of this licence, visit http://creativecommons.org/licenses/by/4.0/.

\section{References}

Papers of particular interest, published recently, have been highlighted as:

- Of importance

•. Of major importance

1. Hill C, Guarner F, Reid G, Gibson GR, Merenstein DJ, Pot B, et al. The International Scientific Association for Probiotics and Prebiotics consensus statement on the scope and appropriate use of the term probiotic. Nat Rev Gastroenterol Hepatol. 2014;11: 506. https://doi.org/10.1038/nrgastro.2014.66.

2. Terciolo C, Dapoigny M, Andre F. Beneficial effects of Saccharomyces boulardii CNCM I-745 on clinical disorders associated with intestinal barrier disruption. Clin Exp Gastroenterol. 2019;12:67-82. https://doi.org/10.2147/ceg.S181590.

3. Vancamelbeke M, Vermeire S. The intestinal barrier: a fundamental role in health and disease. Expert Rev Gastroenterol Hepatol. 2017;11(9):821-34. https://doi.org/10.1080/17474124.2017. 1343143.

4. Bischoff SC, Barbara G, Buurman W, Ockhuizen T, Schulzke J-D, Serino $\mathrm{M}$, et al. Intestinal permeability-a new target for disease prevention and therapy. BMC Gastroenterol. 2014;14:189. https:// doi.org/10.1186/s12876-014-0189-7.
5. Sender R, Fuchs S, Milo R. Revised estimates for the number of human and bacteria cells in the body. PLoS Biol. 2016;14(8): e1002533. https://doi.org/10.1371/journal.pbio.1002533.

6. Press AG, Hauptmann IA, Hauptmann L, Fuchs B, Fuchs M, Ewe $\mathrm{K}$, et al. Gastrointestinal $\mathrm{pH}$ profiles in patients with inflammatory bowel disease. Aliment Pharmacol Ther. 1998;12(7):673-8.

7. Guerra A, Etienne-Mesmin L, Livrelli V, Denis S, Blanquet-Diot S, Alric M. Relevance and challenges in modeling human gastric and small intestinal digestion. Trends Biotechnol. 2012;30(11):591600. https://doi.org/10.1016/j.tibtech.2012.08.001.

8. Ahuja M, Schwartz DM, Tandon M, Son A, Zeng M, Swaim W, et al. Orail-mediated antimicrobial secretion from pancreatic acini shapes the gut microbiome and regulates gut innate immunity. Cell Metab. 2017;25(3):635-46. https://doi.org/10.1016/j.cmet.2017. 02.007.

9. Derrien M, van Hylckama Vlieg JE. Fate, activity, and impact of ingested bacteria within the human gut microbiota. Trends Microbiol. 2015;23(6):354-66. https://doi.org/10.1016/j.tim.2015. 03.002.

10. Zhang C, Derrien M, Levenez F, Brazeilles R, Ballal SA, Kim J, et al. Ecological robustness of the gut microbiota in response to ingestion of transient food-borne microbes. Isme J. 2016;10: 2235-45. https://doi.org/10.1038/ismej.2016.13.

11. Magnúsdóttir S, Ravcheev D, de Crécy-Lagard V, Thiele I. Systematic genome assessment of B-vitamin biosynthesis suggests co-operation among gut microbes. Front Genet. 2015;6:148. https://doi.org/10.3389/fgene.2015.00148 Human gut microbes contain genomic pathways to synthesize $B$ vitamins.

12. Albert MJ, Mathan VI, Baker SJ. Vitamin B12 synthesis by human small intestinal bacteria. Nature. 1980;283(5749):781-2. https:// doi.org/10.1038/283781a0.

13. Yan H, Ajuwon KM. Butyrate modifies intestinal barrier function in IPEC-J2 cells through a selective upregulation of tight junction proteins and activation of the Akt signaling pathway. PLoS One. 2017;12(6):e0179586. https://doi.org/10.1371/journal.pone. 0179586.

14. Zheng L, Kelly CJ, Battista KD, Schaefer R, Lanis JM, Alexeev $\mathrm{EE}$, et al. Microbial-derived butyrate promotes epithelial barrier function through IL-10 receptor-dependent repression of claudin2. J Immunol. 2017;199(8):2976-84. https://doi.org/10.4049/ jimmunol.1700105.

15. Schulthess J, Pandey S, Capitani M, Rue-Albrecht KC, Arnold I, Franchini F, et al. The short chain fatty acid butyrate imprints an antimicrobial program in macrophages. Immunity. 2019;50(2): 432-45.e7. https://doi.org/10.1016/j.immuni.2018.12.018.

16. Sakata T. Stimulatory effect of short-chain fatty acids on epithelial cell proliferation in the rat intestine: a possible explanation for trophic effects of fermentable fibre, gut microbes and luminal trophic factors. Br J Nutr. 1987;58(1):95-103. https://doi.org/10.1079/ BJN19870073.

17. Wu W, Xiao Z, An W, Dong Y, Zhang B. Dietary sodium butyrate improves intestinal development and function by modulating the microbial community in broilers. PLoS One. 2018;13(5): e0197762. https://doi.org/10.1371/journal.pone.0197762.

18. Ford AC, Harris LA, Lacy BE, Quigley EMM, Moayyedi P. Systematic review with meta-analysis: the efficacy of prebiotics, probiotics, synbiotics and antibiotics in irritable bowel syndrome. 2018;48(10):1044-60. https://doi.org/10.1111/apt.15001.

19. Ganji-Arjenaki M, Rafieian-Kopaei M. Probiotics are a good choice in remission of inflammatory bowel diseases: a meta-analysis and systematic review. J Cell Physiol. 2018;233(3):2091-103. https:// doi.org/10.1002/jcp. 25911.

20. Johnston BC, Lytvyn L, Lo CK, Allen SJ, Wang D, Szajewska H, et al. Microbial preparations (probiotics) for the prevention of Clostridium difficile infection in adults and children: An individual patient data Meta-analysis of 6,851 participants. Infect Control 
Hosp Epidemiol. 2018;39(7):771-81. https://doi.org/10.1017/ice. 2018.84.

21. Huang R, Wang $\mathrm{K}, \mathrm{Hu}$ J. Effect of probiotics on depression: a systematic review and meta-analysis of randomized controlled trials. Nutrients. 2016;8(8):483. https://doi.org/10.3390/nu8080483.

22. McFarland LV, Evans CT, Goldstein EJC. Strain-specificity and disease-specificity of probiotic efficacy: a systematic review and meta-analysis. Front Med (Lausanne). 2018;5(124). https://doi. org/10.3389/fmed.2018.00124.

23. Braga VL, Rocha L, Bernardo DD, Cruz CO, Riera R. What do Cochrane systematic reviews say about probiotics as preventive interventions? Sao Paulo Med J. 2017. https://doi.org/10.1590/ 1516-3180.2017.0310241017.

24. Sánchez B, Delgado S, Blanco-Míguez A, Lourenço A, Gueimonde M, Margolles A. Probiotics, gut microbiota, and their influence on host health and disease. Mol Nutr Food Res. 2017;61(1):1600240. https://oi.org/10.1002/mnfr.201600240.

25. Su Y, Chen X, Liu M, Guo X. Effect of three lactobacilli with strainspecific activities on the growth performance, faecal microbiota and ileum mucosa proteomics of piglets. J Anim Sci Biotechnol. 2017;8:52. https://doi.org/10.1186/s40104-017-0183-3.

26.• Yang GY, Yu J, Su JH, Jiao LG, Liu X, Zhu YH. Oral administration of Lactobacillus rhamnosus GG ameliorates Salmonella Infantis-induced inflammation in a pig model via activation of the IL-22BP/IL-22/STAT3 pathway. Front Cell Infect Microbiol. 2017;7:323. https://doi.org/10.3389/fcimb.2017.00323 Proactive administration of probiotics supported the immune system's ability to defend the host against pathogenic microbes.

27. Capurso L. Thirty years of Lactobacillus rhamnosus GG: a review. J Clin Gastroenterol. 2019;53(Suppl 1):S1-s41. https://doi.org/10. 1097/mcg.0000000000001170.

28. Odenwald MA, Turner JR. The intestinal epithelial barrier: a therapeutic target? Nature Reviews Gastroenterology \&Amp. Hepatology. 2016;14:9. https://doi.org/10.1038/nrgastro.2016.169.

29. Niessen CM. Tight junctions/adherens junctions: basic structure and function. J Investig Dermatol. 2007;127(11):2525-32. https:// doi.org/10.1038/sj.jid.5700865.

30. Dignass AU. Mechanisms and modulation of intestinal epithelial repair. Inflamm Bowel Dis. 2001;7(1):68-77. https://doi.org/10. 1097/00054725-200102000-00014.

31. Rao RK, Samak G. Protection and restitution of gut barrier by probiotics: nutritional and clinical implications. Curr Nutr Food Sci. 2013;9(2):99-107.

32. Yi H, Wang L, Xiong Y, Wen X, Wang Z, Yang X, et al. Effects of Lactobacillus reuteri LR1 on the growth performance, intestinal morphology, and intestinal barrier function in weaned pigs. $\mathrm{J}$ Anim Sci. 2018;96(6):2342-51. https://doi.org/10.1093/jas/ sky129 Probiotics maintained growth and structural integrity of small intestine physiology when challenged with an antibiotic.

33. Cui Y, Liu L, Dou X, Wang C, Zhang W, Gao K, et al. Lactobacillus reuteri $\mathrm{ZJ} 617$ maintains intestinal integrity via regulating tight junction, autophagy and apoptosis in mice challenged with lipopolysaccharide. Oncotarget. 2017;8(44):77489-99. https://doi.org/10. 18632/oncotarget.20536.

34. Kim SH, Jeung W, Choi ID, Jeong JW, Lee DE, Huh CS, et al. Lactic acid Bacteria improves Peyer's patch cell-mediated immunoglobulin a and tight-junction expression in a destructed gut microbial environment. J Microbiol Biotechnol. 2016;26(6):1035-45. https://doi.org/10.4014/jmb.1512.12002.

35. Ren C, Dokter-Fokkens J, Figueroa Lozano S, Zhang Q, de Haan $\mathrm{BJ}$, Zhang $\mathrm{H}$, et al. Lactic acid bacteria may impact intestinal barrier function by modulating goblet cells. Mol Nutr Food Res. 2018;62(6):1700572. https://doi.org/10.1002/mnfr.201700572.

36. Chen L, Li H, Li J, Chen Y, Yang Y. Lactobacillus rhamnosus GG treatment improves intestinal permeability and modulates microbiota dysbiosis in an experimental model of sepsis. Int $\mathrm{J}$ Mol Med. 2019;43(3):1139-48. https://doi.org/10.3892/ijmm. 2019.4050 .

37. Dukowicz AC, Lacy BE, Levine GM. Small intestinal bacterial overgrowth: a comprehensive review. Gastroenterol Hepatol (N Y). 2007;3(2):112-22.

38. Quigley EM, Fried M, Gwee KA, Khalif I, Hungin AP, Lindberg G, et al. World gastroenterology organisation global guidelines irritable bowel syndrome: a global perspective update September 2015. J Clin Gastroenterol. 2016;50(9):704-13. https://doi.org/10.1097/ mcg.0000000000000653.

39. Saffouri GB, Shields-Cutler RR, Chen J, Yang Y, Lekatz HR, Hale VL, et al. Small intestinal microbial dysbiosis underlies symptoms associated with functional gastrointestinal disorders. Nat Commun. 2019;10(1):2012. https://doi.org/10.1038/s41467-019-09964-7.

40. Fine KD, Schiller LR. AGA technical review on the evaluation and management of chronic diarrhea. Gastroenterology. 1999;116(6): 1464-86. https://doi.org/10.1016/S0016-5085(99)70513-5.

41. Bouhnik Y, Alain S, Attar A, Flourie B, Raskine L, Sanson-Le Pors $\mathrm{MJ}$, et al. Bacterial populations contaminating the upper gut in patients with small intestinal bacterial overgrowth syndrome. Am J Gastroenterol. 1999;94(5):1327-31. https://doi.org/10.1111/j. 1572-0241.1999.01016.x.

42. Gatta L, Scarpignato C. Systematic review with meta-analysis: rifaximin is effective and safe for the treatment of small intestine bacterial overgrowth. Aliment Pharmacol Ther. 2017;45(5):604 16. https://doi.org/10.1111/apt.13928.

43. Barkin JA, Keihanian T, Barkin JS, Antequera CM, Moshiree B. Preferential usage of rifaximin for the treatment of hydrogenpositive small intestinal bacterial overgrowth. Rev Gastroenterol Peru. 2019;39(2):111-5.

44. Grace E, Shaw C, Whelan K, Andreyev HJN. Review article: small intestinal bacterial overgrowth - prevalence, clinical features, current and developing diagnostic tests, and treatment. Aliment Pharmacol Ther. 2013;38(7):674-88. https://doi.org/10.1111/apt. 12456 .

45. Zhong C, Qu C, Wang B, Liang S, Zeng B. Probiotics for preventing and treating small intestinal bacterial overgrowth: a meta-analysis and systematic review of current evidence. J Clin Gastroenterol. 2017;51(4):300-11. https://doi.org/10.1097/mcg. 0000000000000814.

46. Kumar K, Saadi M, Ramsey FV, Schey R, Parkman HP. Effect of Bifidobacterium infantis 35624 (align) on the lactulose breath test for small intestinal bacterial overgrowth. Dig Dis Sci. 2018;63(4): 989-95. https://doi.org/10.1007/s10620-018-4945-3.

47. Aziz I, Tornblom H, Simren M. Small intestinal bacterial overgrowth as a cause for irritable bowel syndrome: guilty or not guilty? Curr Opin Gastroenterol. 2017;33(3):196-202. https://doi.org/10. 1097/mog.0000000000000348.

48. Stanghellini V. Functional dyspepsia and irritable bowel syndrome: beyond Rome IV. Dig Dis (Basel, Switzerland). 2017;35(Suppl 1): 14-7. https://doi.org/10.1159/000485408.

49. Principi N, Cozzali R, Farinelli E, Brusaferro A, Esposito S. Gut dysbiosis and irritable bowel syndrome: the potential role of probiotics. J Infect. 2018;76(2):111-20. https://doi.org/10.1016/j. jinf.2017.12.013.

50. Catinean A, Neag AM, Nita A, Buzea M, Buzoianu AD. Bacillus spp. spores-A promising treatment option for patients with irritable bowel syndrome. Nutrients. 2019;11(9). https://doi.org/10.3390/ nu11091968.

51. Dunlop SP, Hebden J, Campbell E, Naesdal J, Olbe L, Perkins AC, et al. Abnormal intestinal permeability in subgroups of diarrheapredominant irritable bowel syndromes. Am J Gastroenterol. 2006;101(6):1288-94. https://doi.org/10.1111/j.1572-0241.2006. 00672.x. 
52. Goswami P, Das P, Verma AK, Prakash S, Das TK, Nag TC, et al. Are alterations of tight junctions at molecular and ultrastructural level different in duodenal biopsies of patients with celiac disease and Crohn's disease? Virchows Arch. 2014;465(5):521-30. https:// doi.org/10.1007/s00428-014-1651-1.

53. Dunne WT, Cooke WT, Allan RN. Enzymatic and morphometric evidence for Crohn's disease as a diffuse lesion of the gastrointestinal tract. Gut. 1977;18(4):290-4. https://doi.org/10.1136/gut.18.4.290.

54.• Pérez-Torras S, Iglesias I, Llopis M, Lozano JJ, Antolín M, Guarner $\mathrm{F}$, et al. Transportome profiling identifies profound alterations in Crohn's disease partially restored by commensal bacteria. J Crohn's Colitis. 2016;10(7):850-9. https://doi.org/10.1093/eccojcc/jjw042 Dysregulated transporters are found in Crohn's disease, and beneficial commensal gut bacteria can improve function.

55. Wang W, Chen L, Zhou R, Wang X, Song L, Huang S, et al. Increased proportions of Bifidobacterium and the Lactobacillus group and loss of butyrate-producing bacteria in inflammatory bowel disease. J Clin Microbiol. 2014;52(2):398-406. https://doi. org/10.1128/jcm.01500-13.

56. Gevers D, Kugathasan S, Denson LA, Vazquez-Baeza Y, Van Treuren W, Ren B, et al. The treatment-naive microbiome in newonset Crohn's disease. Cell Host Microbe. 2014;15(3):382-92. https://doi.org/10.1016/j.chom.2014.02.005 Mucosal sampling of Crohn's disease reveals a distrinct microbial profile with a reduction in butyrate producing bacteria.

57. Wright EK, Kamm MA, Teo SM, Inouye M, Wagner J, Kirkwood CD. Recent advances in characterizing the gastrointestinal microbiome in Crohn's disease: a systematic review. Inflamm Bowel Dis. 2015;21(6):1219-28. https://doi.org/10.1097/mib. 0000000000000382.

58. De Cruz P, Kang S, Wagner J, Buckley M, Sim WH, Prideaux L, et al. Association between specific mucosa-associated microbiota in Crohn's disease at the time of resection and subsequent disease recurrence: a pilot study. J Gastroenterol Hepatol. 2015;30(2): 268-78. https://doi.org/10.1111/jgh.12694.

59. Geirnaert A, Calatayud M, Grootaert C, Laukens D, Devriese S, Smagghe G, et al. Butyrate-producing bacteria supplemented in vitro to Crohn's disease patient microbiota increased butyrate production and enhanced intestinal epithelial barrier integrity. Sci Rep. 2017;7(1):11450. https://doi.org/10.1038/s41598-017-11734-8.

60. Derwa Y, Gracie DJ, Hamlin PJ, Ford AC. Systematic review with meta-analysis: the efficacy of probiotics in inflammatory bowel disease. Aliment Pharmacol Ther. 2017;46(4):389-400. https:// doi.org/10.1111/apt.14203.

61. Langella P, Guarner F, Martín R. Editorial: next-generation probiotics: from commensal bacteria to novel drugs and food supplements. Front Microbiol. 2019;10(1973). https://doi.org/10.3389/ fmicb.2019.01973.

62. Sjöberg F, Barkman C, Nookaew I, Östman S, Adlerberth I, Saalman R, et al. Low-complexity microbiota in the duodenum of children with newly diagnosed ulcerative colitis. PLoS One. 2017;12(10):e0186178. https://doi.org/10.1371/journal.pone. 0186178.

63. Taylor L, Almutairdi A, Shommu N, Fedorak R, Ghosh S, Reimer RA, et al. Cross-sectional analysis of overall dietary intake and
Mediterranean dietary pattern in patients with Crohn's disease. Nutrients. 2018;10(11). https://doi.org/10.3390/nu10111761.

64. Martinez-Guryn K, Hubert N, Frazier K, Urlass S, Musch MW, Ojeda $\mathrm{P}$, et al. Small intestine microbiota regulate host digestive and absorptive adaptive responses to dietary lipids. Cell Host Microbe. 2018;23(4):458-69.e5. https://doi.org/10.1016/j.chom. 2018.03.011.

65. Fan P, Liu P, Song P, Chen X, Ma X. Moderate dietary protein restriction alters the composition of gut microbiota and improves ileal barrier function in adult pig model. Sci Rep. 2017;7:43412. https://doi.org/10.1038/srep43412.

66. Nettleton JE, Reimer RA, Shearer J. Reshaping the gut microbiota: impact of low calorie sweeteners and the link to insulin resistance? Physiol Behav. 2016;164(Pt B):488-93. https://doi.org/10.1016/j. physbeh.2016.04.029.

67. Chassaing B, Van de Wiele T, De Bodt J, Marzorati M, Gewirtz AT. Dietary emulsifiers directly alter human microbiota composition and gene expression ex vivo potentiating intestinal inflammation. Gut. 2017;66(8):1414-27. https://doi.org/10.1136/gutjnl-2016313099.

68. Tuohy KM, Conterno L, Gasperotti M, Viola R. Up-regulating the human intestinal microbiome using whole plant foods, polyphenols, and/or fiber. J Agric Food Chem. 2012;60(36):8776-82. https://doi.org/10.1021/jf2053959.

69. Tomova A, Bukovsky I, Rembert E, Yonas W, Alwarith J, Barnard ND, et al. The effects of vegetarian and vegan diets on gut microbiota. Front Nutr. 2019;6:47. https://doi.org/10.3389/fnut.2019. 00047.

70. Zinocker MK, Lindseth IA. The Western diet-microbiome-host interaction and its role in metabolic disease. Nutrients. 2018;10(3). https://doi.org/10.3390/nu10030365.

71. Benjamin J, Makharia GK, Kalaivani M, Joshi YK. Nutritional status of patients with Crohn's disease. Indian J Gastroenterol. 2008;27(5):195-200.

72. Fabisiak N, Fabisiak A, Watala C, Fichna J. Fat-soluble vitamin deficiencies and inflammatory bowel disease: systematic review and meta-analysis. J Clin Gastroenterol. 2017;51(10):878-89. https://doi.org/10.1097/mcg.0000000000000911.

73. Tuddenham S, Sears CL. The intestinal microbiome and health. Curr Opin Infect Dis. 2015;28(5):464-70. https://doi.org/10.1097/ QCO.0000000000000196.

74. Blutt SE, Crawford SE, Ramani S, Zou WY, Estes MK. Engineered human gastrointestinal cultures to study the microbiome and infectious diseases. Cell Mol Gastroenterol Hepatol. 2017;5(3):241-51. https://doi.org/10.1016/j.jcmgh.2017.12.001.

75. Wang L, Llorente C, Hartmann P, Yang A-M, Chen P, Schnabl B. Methods to determine intestinal permeability and bacterial translocation during liver disease. J Immunol Methods. 2015;421:44-53. https://doi.org/10.1016/j.jim.2014.12.015.

76. Dorshow RB, Johnson JR, Debreczeny MP, Riley IR, Shieh J-J, Rogers TE et al. Noninvasive point-of-care measurement of gastrointestinal permeability. SPIE BiOS SPIE; 2019.

Publisher's Note Springer Nature remains neutral with regard to jurisdictional claims in published maps and institutional affiliations. 